\title{
Tools for validating and creating reliable fault models
}

\author{
J Danielson BGC Engineering Inc., Canada \\ D Kinakin BGC Engineering Inc., Canada \\ I Stilwell BGC Engineering Inc., Canada
}

\begin{abstract}
Reliable 3D fault models are critically important for open pit slope design and slope stability assessment. Fault-influenced slope failures are major geotechnical hazards which frequently result in production slowdowns and require mitigation through design changes or depressurisation. Despite its importance in assessing slope stability, 3D fault model development is often left to the mine geologists or external geological consultants, whose focus is commonly the distribution of ore, not geotechnical hazard. This study presents a data-driven, semi-automated method for validating, identifying, and mapping faults that can influence slope stability. The tools are developed using the Python programming language's freely available scientific computing libraries. These scalable libraries are used to leverage and visualise massive datasets, like blasthole drilling data or rock quality designation (RQD) to assess and improve fault models. Two case studies are provided to illustrate the application of the tools.
\end{abstract}

Keywords: fault modelling, pit slope instabilities, rock strength distribution, data visualisation

\section{Introduction}

Reliable fault models are critical to designing open pits and assessing slope stability. Most slope failures at open pit mines involve one or more faults; in these cases, predicting or back-analysing behaviour requires an understanding of the location, orientation, and character of the faults involved.

Mine fault models often focus on faults related to ore distribution, but offsets in ore distribution do not necessarily correspond with brittle faults, and geotechnically important faults may not control ore. Geotechnical practitioners should aim to identify all faults that represent zones of weakness within the rock mass. These zones of weakness can be observed as a decrease in rock quality, an increase in fracture frequency, evidence of a thick gouge core, or a decrease in strength. Identifying and assessing these faults requires finding these characteristics within the available data.

Spatial mathematics were previously laborious and difficult. For this reason, when geotechnical and mine engineers do try to identify or validate faults, the work often involves simply looking at core photos or spot-checking rock quality in one or two drillholes. Although looking at core photos and spot-checking specific drillholes is important in understanding a rock mass, this type of validation is subjective and should not be the only method of geotechnically validating faults.

Recent software advancements allow a more quick, robust, and data-driven method of geotechnically validating faults. With enough data, even very 'noisy' datasets can be used to map and validate faults using signed distance calculations, data density plots, and semi-automated workflows. These tools are demonstrated to be applicable using two datasets, specific energy and rock quality designation (RQD).

\section{$2 \quad$ Methodology}

\subsection{Fault zone architecture}

To identify, map, and validate brittle faults from geotechnical data, one needs first to understand what faults are and how they are typically expressed in the data. Then, one can compare this conceptual model of what 
a fault is to the available data and determine whether the evidence supports or contradicts a fault's current interpretation.

A brittle fault is a discontinuity along which displacement has occurred, causing strain, fracturing, and damage to the surrounding rock mass. Major fault zones typically include the following characteristics (Figure 1):

- A relatively (in comparison to the fault core) wide damage zone, where the frequency of fractures, shears, and minor or secondary faults increases approaching the fault core.

- A fault core, which includes discrete shear zones, fault gouge, cataclasites, and/or fault breccia.

- A parallel or subparallel set of faults and fractures. The stress regimes which cause large faults to form in certain orientations can also cause smaller faults to form at those orientations.

Evidence for a major fault should therefore include at least one of the following:

- Increasing fracture frequency (measured directly as fractures per metre or through RQD).

- An increasing frequency of faults and shears.

- Decreasing rock or rock mass quality.

- Decreasing strength, possibly a sharp decrease near or in the fault core.

Faults with the potential to be geotechnically important should be tested against available evidence. This paper presents a simple and quick method of doing so: the signed distance plot.

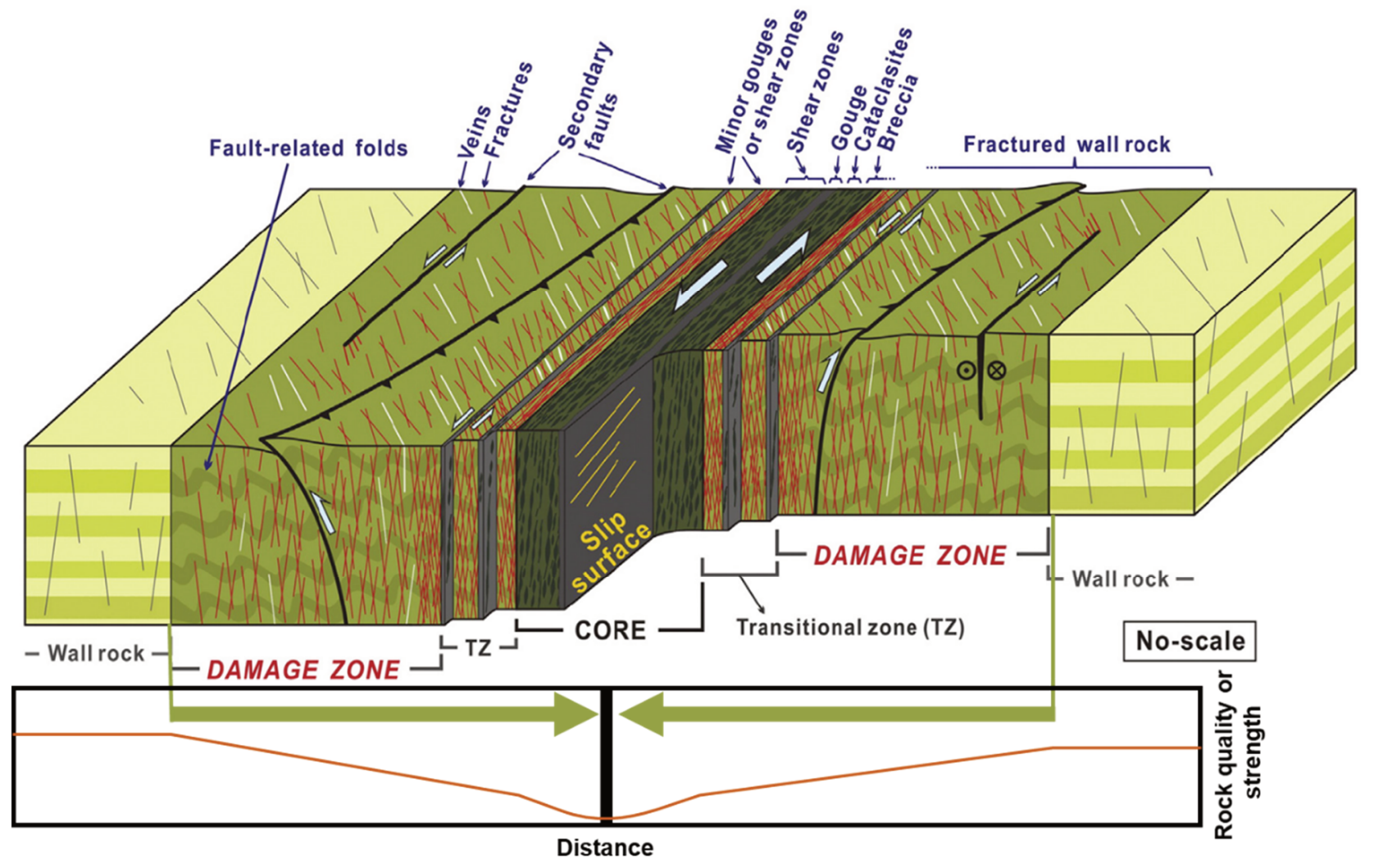

Figure 1 Conceptual diagram of a fault zone (after Choi et al. 2016) 


\subsection{Candidate faults}

The tools presented in the current work have been developed to compare and validate 3D surfaces representing 'candidate faults' against geotechnical data from drillholes. The candidate faults may come from many sources, including:

- Picks from drillholes.

- Topographic lineaments.

- Geophysical lineaments.

- Geological unit contacts.

- Projections from outcrop mapping.

The efficiency of the presented tools allows large numbers of candidate faults to be validated against the available geotechnical datasets. In this regard, the tools presented in this paper support the testing of geological hypotheses and consideration of alternate fault interpretations.

\subsection{The signed distance plot}

Given that a fault can only impact stability if it impacts the geotechnical properties of a rock, a geotechnically important fault should be detectable in geotechnical data. Validation of a candidate fault should therefore involve looking at how geotechnical data change approaching a fault. These data can include fracture frequency or quality measurements from core logging or photogrammetry mapping, or unconventional measures of strength like specific energy. A rudimentary way to validate a candidate fault with geotechnical data is to compare average geotechnical parameters within a certain distance of the fault zone, to average geotechnical parameters farther from the fault zone. But this type of comparison doesn't show data variability, whether the difference is significant, or the character of the change in geotechnical parameters. A much more robust method is to calculate signed distance between the geotechnical dataset and the fault surface to be validated, and then to plot the dataset against that signed distance.

Signed distance is simply distance with a sign indicating direction, which can be calculated using a number of programs (e.g. CloudCompare 2019) or Python libraries (e.g. Trimesh (Dawson-Haggerty 2019)). Signed distance between a point and a triangulated mesh is a common calculation in computer graphics, because triangulated meshes are by far the most popular method of representing 3D objects (Bærentzen \& Aanæs 2005). A number of efficient techniques have been developed to quickly calculate signed distance (e.g. Barill et al. 2018). In this study, distance is calculated as the minimum distance between a point and the analysed fault surface (modelled as a triangulated mesh), and the sign is calculated as toward the hanging wall direction (positive) or the footwall direction (negative).

Once the signed distance from a fault is calculated for a geotechnical dataset, the dataset can be plotted with magnitude on the $y$ axis and distance on the $x$ axis. Plotted data can include a binned median or average (similar to the conceptual plot at the bottom of Figure 1), or use the more sophisticated hexbin technique, which divides the graph space into equally sized hexagonal bins, then assigns a colour based on how many data points fall within each bin, or both. A hexbin plot is essentially a $2 \mathrm{D}$ version of the $1 \mathrm{D}$ histogram, which allows a comparison of the parameter distribution against another parameter (in this case, distance).

In addition to showing whether rock quality decreases near a fault, signed distance plots also show whether rock properties differ on either side of the fault. If the fault is a geotechnical unit boundary, and rock quality differs between the hanging wall and footwall, this will be visible in the signed distance analysis of the fault. Signed distance plots can also show the presence and location of faults parallel to the fault surface analysed. Examples are shown in Sections 3 and 5. Signed distance plots can be generated for faults of any geometry; they do not require a planar input. 


\section{4 'Fault finder': using signed distance to locate and map faults}

Signed distance plots show not only whether a candidate fault (represented by a change in geotechnical parameters) exists at a given location, but also if the fault exists at some parallel distance offset from the queried location or candidate 3D surface. Therefore, a collection of plots generated for every possible fault orientation could theoretically be used to identify and locate all faults of all orientations within the dataset. Of course, even limiting this exercise to one plot per degree, this would require the generation of 32,400 plots $\left(360^{\circ} \times 90^{\circ}\right)$. Several years ago, this would have been a near-impossible task. Today, freely available Python libraries allow relatively quick and easy computation and plotting of this process.

The process described below generates signed distance plots for hypothetical fault planes of all orientations and returns the largest trough in the geotechnical parameter considered at each orientation. The largest trough at each orientation is plotted on a stereonet. Low values of strength or RQD on the resultant stereonets generally indicate faults.

Although the steps of this brute force algorithm (below) require significant computation, the first six of the seven-step process can be automated:

1. Import the geotechnical dataset (examples: specific energy or RQD).

2. Import a planar mesh.

3. Rotate the plane through a very large range of orientations.

4. For each possible orientation combination, calculate the binned median (or mean) geotechnical parameter for given signed distance bins, and return the lowest value (equivalent to the largest trough in the signed distance plot).

5. Translate the rotated plane by the distance at which the trough is located.

6. Collate and plot the results in stereonet projection space.

7. Identify local lows in the geotechnical parameter analysed and investigate the results.

The time required to generate results of steps 1-6 depends on the size of the dataset analysed and the resolution desired, but useable results can typically be generated in less than five minutes. Example results from this process are discussed in Sections 3.3 and 4.3.

The 'fault finder' algorithm has significant limitations. First, the algorithm is most effective at identifying faults that are approximately planar at the scale assessed. If rock quality doesn't vary with distance from the assessed plane significantly enough it will be indistinguishable from the background variability of the dataset, and the results will not indicate the presence of a fault. This limitation can be partially overcome in a folded geological setting by dividing the input dataset into smaller spatial subsections.

A second limitation is that this method gives the clearest results given a large input dataset with a relatively consistent data density. The minimum drillhole density required to produce useful results likely depends on the variability of the rock mass; the thickness, persistence, and planarity of faults cross-cutting the study area; and the difference in rock quality between faulted and un-faulted rock. A small geotechnical drilling dataset with 5 to 15 drillholes drilled over a $500 \times 500 \mathrm{~m}$ area likely does not have the data density required to produce meaningful results using this method, and it is not possible to map faults from geotechnical data in zones where no geotechnical data are available.

Despite these limitations, because this method relies on data quantity over data quality, even relatively noisy datasets such as specific energy (see Section 3: case study 1) and exploration RQD data (see Section 4: case study 2) can be used as inputs. 


\section{$3 \quad$ Case study 1: validating and mapping faults from a specific energy dataset collected at a Canadian copper mine}

\subsection{The specific energy dataset}

Case study 1 uses a specific energy dataset from a mine in British Columbia, Canada to validate and map faults. Specific energy, the energy required to excavate a unit volume of rock, is a measure of rock strength that can be collected from blasthole drilling (Teale 1965).

The specific energy data used in case study 1 was measured automatically during blasthole drilling for use in optimising blasts (powder factor, etc.). Because specific energy is a particularly noisy dataset, it is not often used for geotechnical design. At this mine, specific energy is calculated as:

$$
S E=\frac{F}{A}+\frac{2 \pi}{A}\left(\frac{N T}{u}\right)
$$

where:

$\mathrm{F} \quad=$ is force or thrust.

$\mathrm{A}=$ bit area.

$\mathrm{T}=$ torque.

$\mathrm{N} \quad=$ rotation speed .

$\mathrm{U} \quad=$ penetration rate.

Specific energy can be described in terms of $\mathrm{MJ} / \mathrm{m}^{3}$, or, because one joule is equivalent to one newton metre, in terms of $\mathrm{MNm} / \mathrm{m}^{3}$ or more simply MPa. Specific energy is frequently described in terms of MPa because it is used as a measure of rock strength (Richard et al. 2012), and because $\mathrm{MJ} / \mathrm{m}^{3}$ and $\mathrm{MPa}$ are dimensionally identical.

Because blastholes are drilled wherever mining is carried out, specific energy datasets can be very large. The dataset used in this paper includes data from over 200,000 blast drillholes and includes over 10 million datapoints. The blastholes in this dataset have an average length of just $17 \mathrm{~m}$, but because blastholes are drilled within every bench mined, the dataset has a depth that spans the entire pit slope.

Figure 2 shows the specific energy dataset used in case study 1 along with a photogrammetry model of the open pit. Data are shown as a point cloud coloured by specific energy magnitude. Blue points indicate specific energy values of $60 \mathrm{MPa}$ or greater, and green, yellow, and orange points indicate specific energy values of $40 \mathrm{MPa}$ or lower, values associated with faults and fault damage zones in the pit. Filtering methods like the bilateral filter (Paris \& Durand 2009) can be used to reduce the noise in the specific energy dataset (Danielson et al. 2017), making the faults that crosscut the open pit much more clearly visible (example shown on Figure 2c); but the current paper uses the unfiltered dataset to demonstrate the robustness of the techniques described above. 

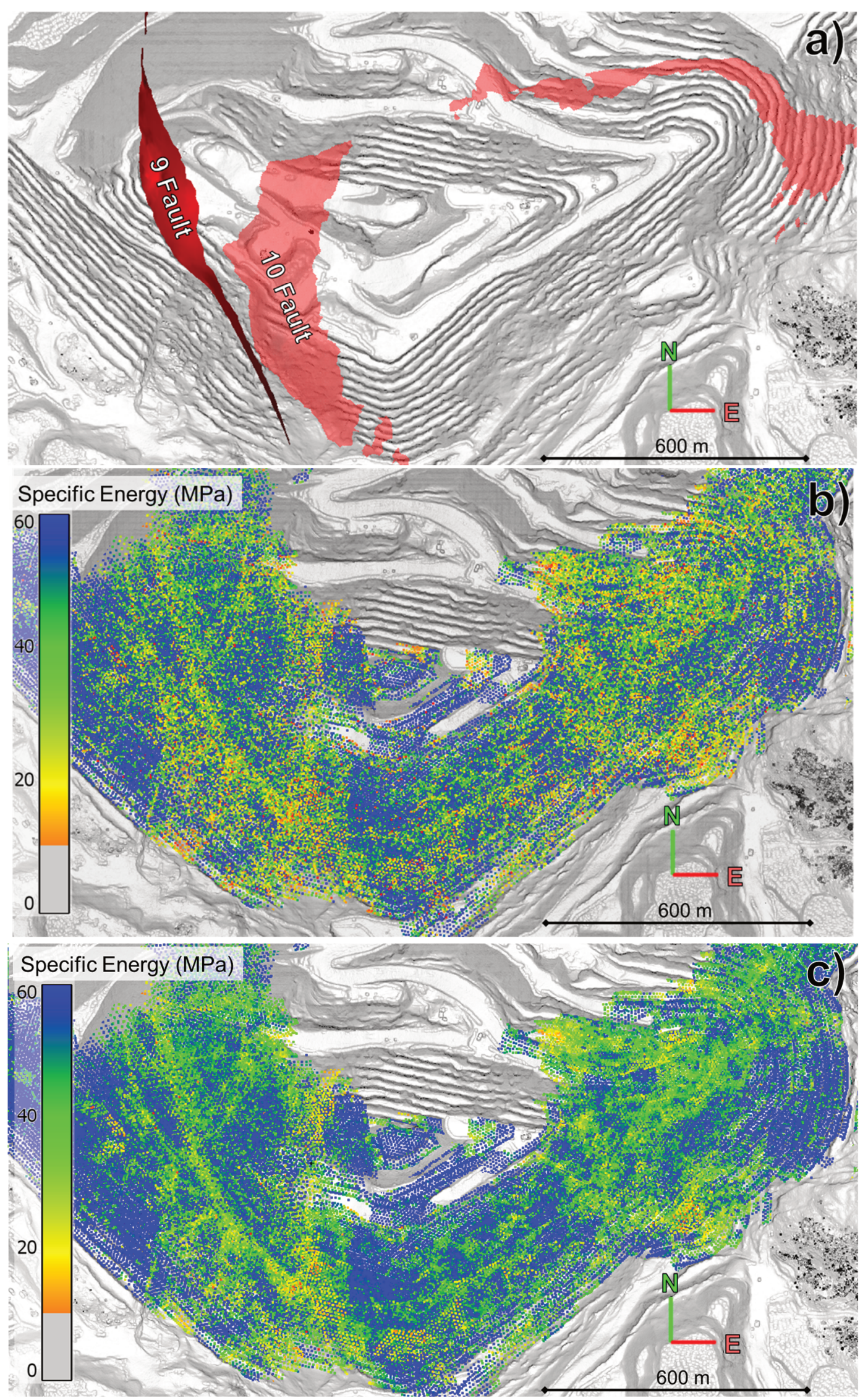

Figure 2 (a) Unmanned aerial vehicle photogrammetry of the open pit; (b) The specific energy dataset; and (c) The specific energy dataset after applying a bilateral filter (after Danielson 2018) 


\subsection{Using signed distance to validate a fault model}

Given that specific energy is a measure of rock strength, and faults weaken a rock mass, the specific energy-signed distance plot for a valid fault should indicate a drop in specific energy approaching the fault. Figure 3 shows an example signed distance plot for a major fault. As expected, strength decreases sharply approaching the fault centre. This example also highlights the usefulness of the hexbin plotting method: one can see that not only the median specific energy drops approaching the fault, but the entire distribution shifts, and ' $v$ ' shapes are visible not only in the most data-dense bins but also in the less dense bins (Figure 3). A poorly mapped fault or a fault orientation with no evidence appears as an approximately straight line (Figure 4) in the specific energy data.

One benefit of the signed distance plot is that because distance is signed, and not absolute, the plot also shows the rock quality at specified distances from the structure. Therefore, if the orientation of the tested fault is correct, but the fault was modelled in the incorrect location, the fault will still be visible in the signed distance plot. The plot will show that the fault does exist and will also show the distance by which the modelled fault is incorrect. Figure 5 shows an example where a fault was modelled with the correct orientation but offset by $30 \mathrm{~m}$ in the hanging wall direction from the actual structure. The ability of signed distance plots to show faults offset from the queried location is discussed further in Section 3.3.

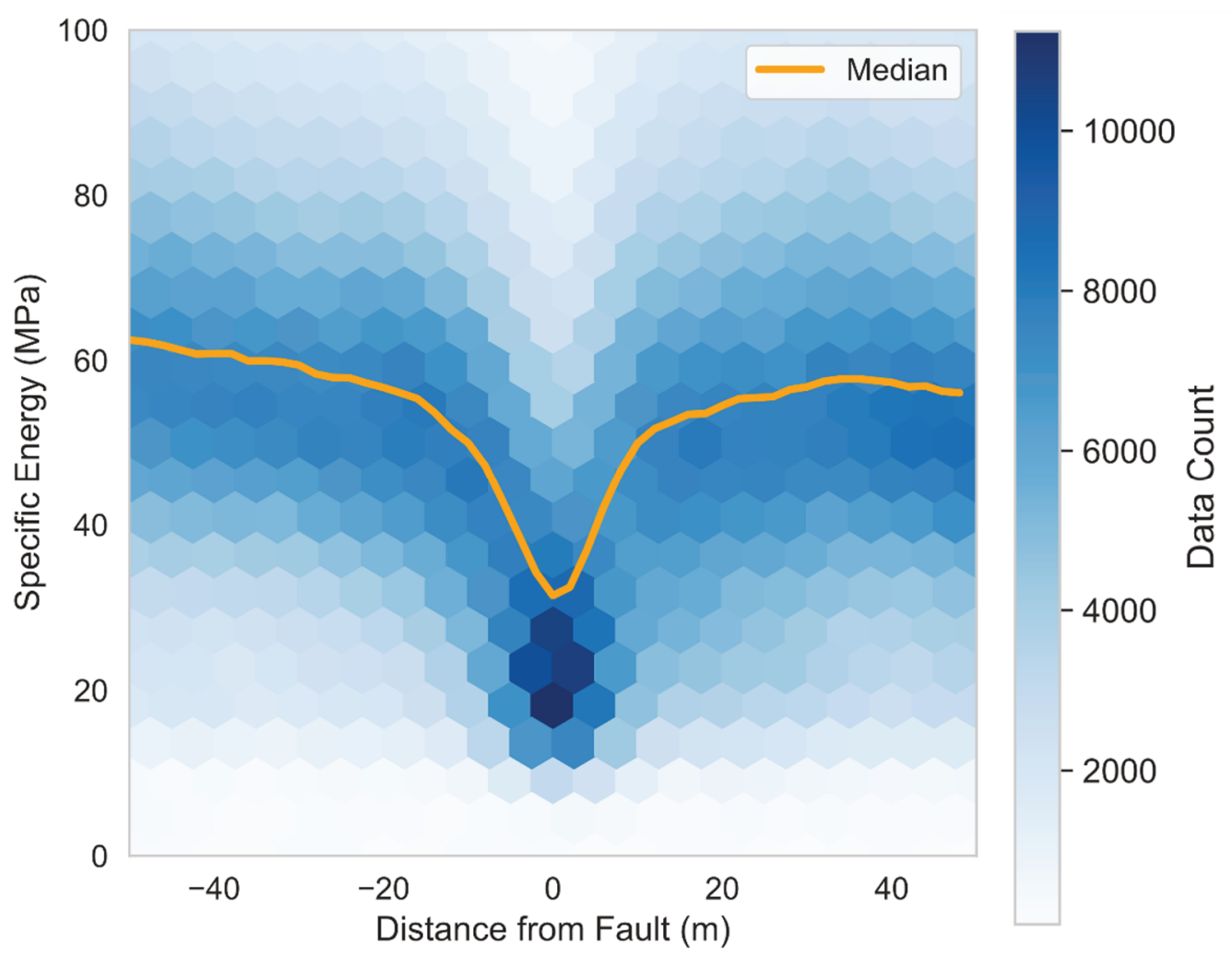

Figure 3 Specific energy versus distance from a known major fault 


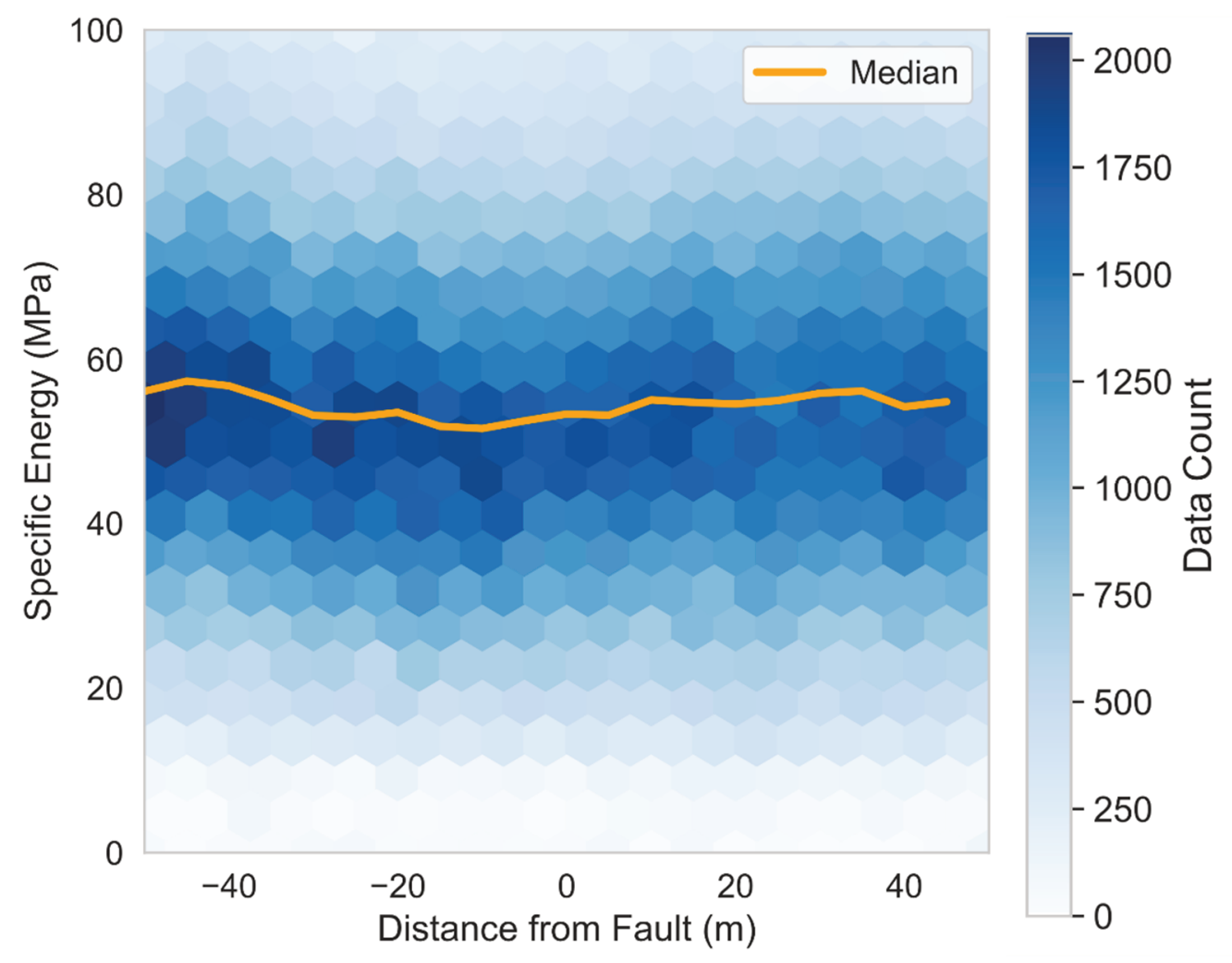

Figure 4 Specific energy versus distance from a very poorly mapped fault. Note that there is no clear relationship between distance from the fault and specific energy

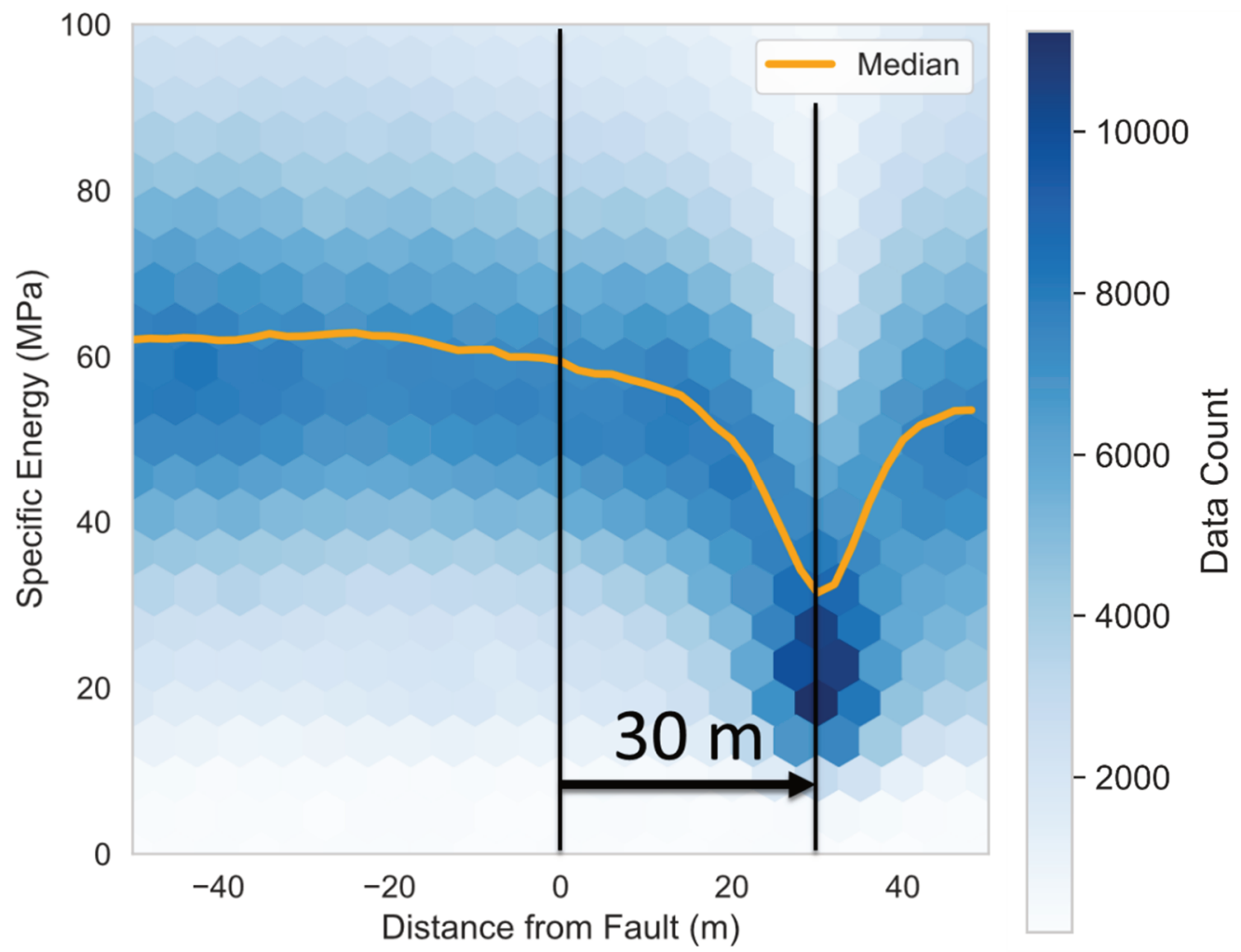

Figure 5 Specific energy versus distance from a plane offset $30 \mathrm{~m}$ from a major fault. Note that although the location queried is incorrect, the fault is still clearly visible in data 


\subsection{Using signed distance to locate and map faults with specific energy data}

The process described in Section 2.4, where a plane is rotated through all possible orientations to find troughs in the resultant signed distance plots, can be used with any geotechnical dataset, including specific energy. An example of this process uses a selection of the specific energy dataset (Figure 6a) as input and plots the minimum of the mean specific energy binned by signed distance on a stereonet for every possible orientation. Stereonets are procedurally generated using the mplstereonet (Kington 2019) Python library. The resulting stereonet (Figure 6b) shows two major lows in specific energy. Exporting the planes at these orientations (Figure 6c) shows they match very closely with two faults running through the specific energy data (Figure 6d).
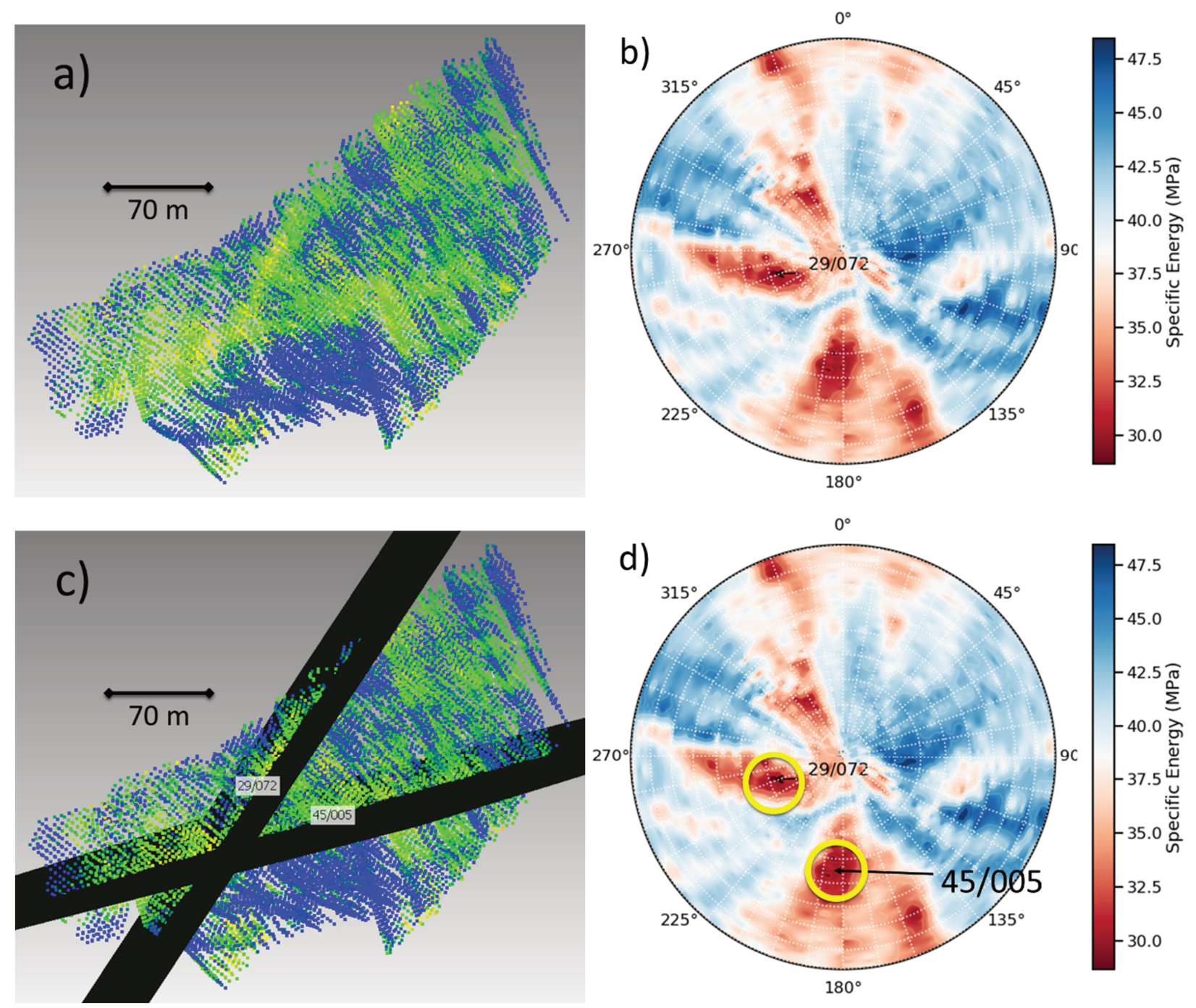

Figure 6 Algorithmically finding and mapping faults in the specific energy dataset. (a) The selection of specific energy data selected for analysis; (b) The results of the brute force algorithm plotted in stereonet projection; (c) The planes generated by the algorithm; and (d) The local lows in specific energy highlighted on the stereonet 


\section{$4 \quad$ Case study 2: validating and mapping faults from exploration drilling data at an African mine}

\subsection{The RQD dataset}

The second case study involves analysing RQD (Deere \& Deere 1988) from exploration core logging data collected at a mine in Africa. RQD is one of the most frequently collected measures of rock quality. It is also one of the noisiest, most inconsistently measured, and least reliable measures of rock quality (Pells et al. 2017). The current study considers an RQD dataset from exploration drilling, collected by different technicians over the span of more than 10 years. The dataset includes RQD data from 157 drillholes, drilled at uneven spacing but with very similar orientations (Figure 7).

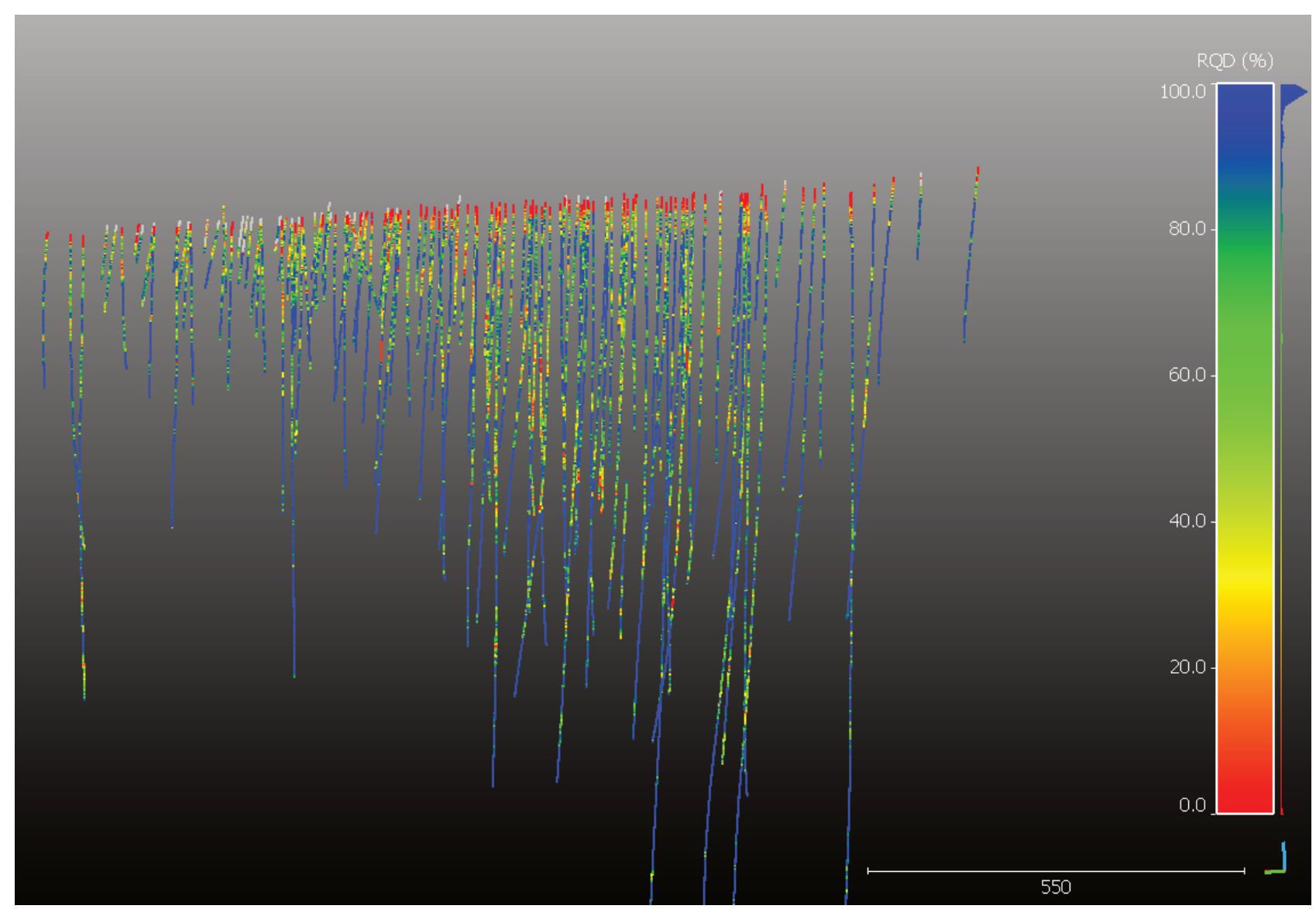

Figure 7 The RQD dataset used for the current study

\subsection{Using signed distance to validate a major fault using RQD data}

The signed distance technique also works with noisy RQD data. Plotting RQD from the dataset described above against distance from a known fault (Figure 8), although less elegant than the specific energy plot, shows a clear drop in binned median RQD, and a higher density of lower-RQD data approaching the fault.

This technique is not only useful for noisy datasets-it works more effectively with better-behaved datasets, and can be used with a variety of fault indicators. Other fault indicators that may be useful are the quantity of logged faults divided by the total length of logged core in a given bin, gouge length divided by length of logged core, fracture frequency, strength, or geological strength index (GSI). The noisier the dataset, the larger the dataset required for the signal to outweigh the noise. 


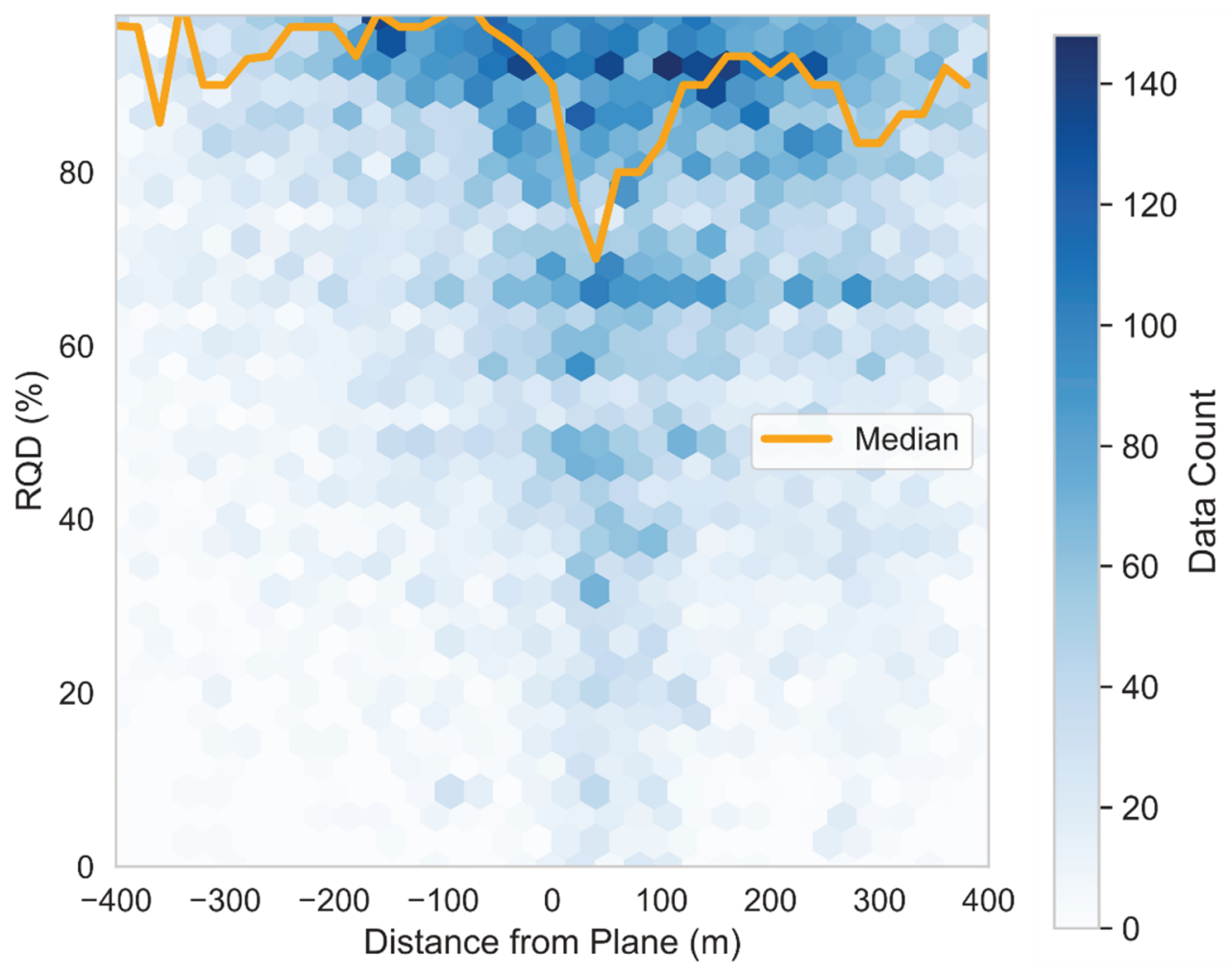

Figure 8 RQD (\%) versus distance from a known fault

\subsection{Using signed distance to locate a major fault with RQD data}

The second example of this process uses the entire RQD dataset described above and plots the lowest binned median RQD at each orientation. The results (Figure 9) show several lows in RQD, the most prominent aligning with a planar contact between two units which had not been included in the 3D fault model and had not previously been identified as a major fault. Inspection of geotechnical logs and core photos was used to confirm the fault zone's existence.

This process works best for identifying planar faults, but faults do not have to be perfectly planar to show a statistically significant drop in specific energy or RQD.

Not all faults will be evidenced in RQD. If loggers are not properly applying the soundness criteria (which excludes rock $<R 1$ from being included in the sum of intact rock lengths greater than $10 \mathrm{~cm}$ ), if the fault doesn't have a surrounding damage zone, or if the fault has a very limited thickness it may be imperceptible from noise. Other geotechnical parameters also need to be considered. RQD and specific energy are presented here because they're relatively poor indicators of faulting, and yet return clear results. 

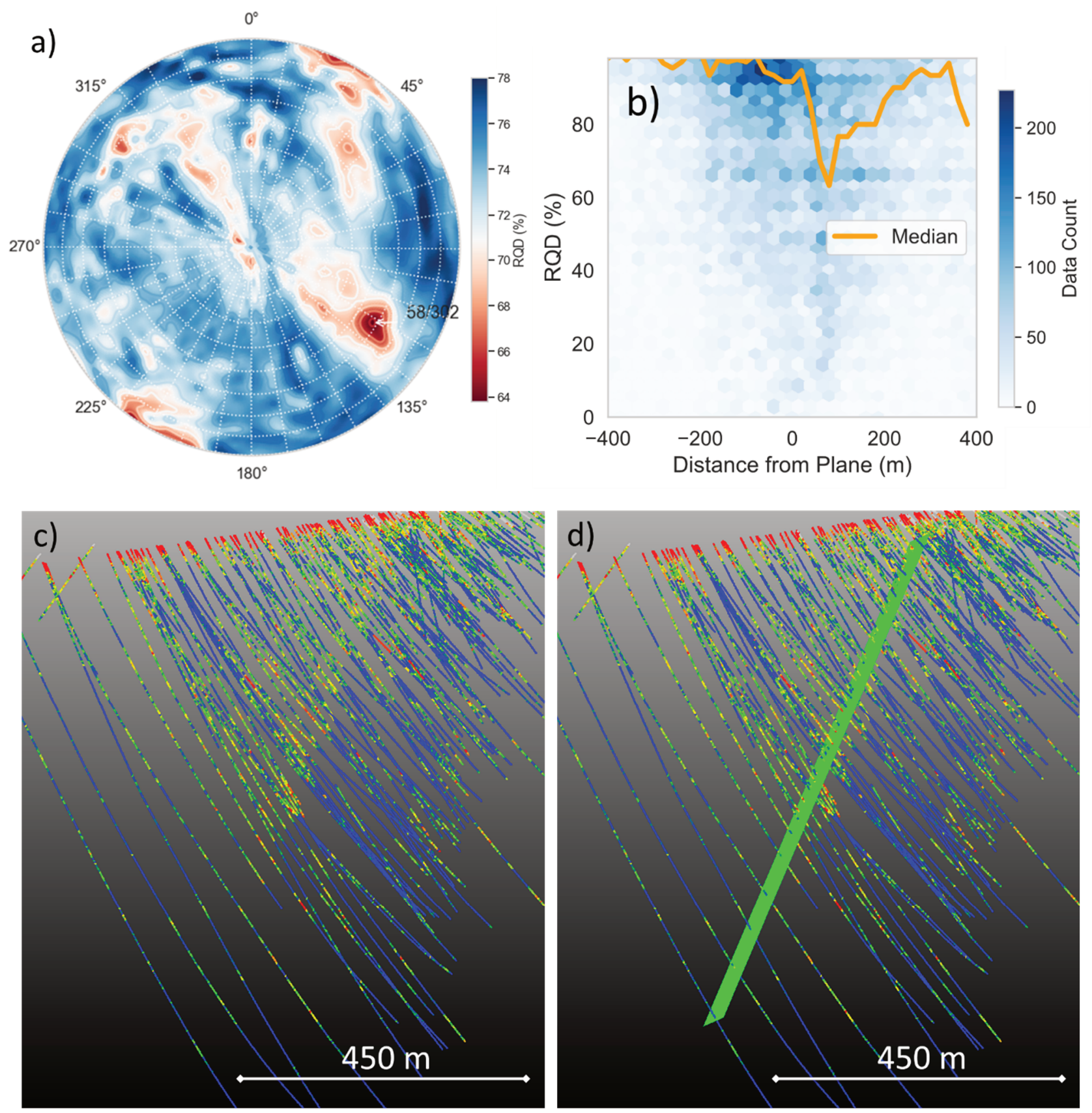

Figure 9 Algorithmically finding and mapping faults in the RQD dataset. (a) Results plotted on a stereonet; (b) The signed distance plot of the plane oriented at $58^{\circ} / 302^{\circ}$ (dip/dip direction); (c) The RQD dataset; and (d) The RQD dataset with the plane generated by the brute force algorithm

\section{Conclusion}

Faults are one of the most critical factors controlling slope stability. Pit slope design and slope stability analyses will be more realistic if they use realistic, well-evidenced faults as inputs.

A fault can only affect stability if it has a different geotechnical character than the surrounding rock. Plotting fault indicators, including fracture frequency from drillcore or photogrammetry, rock strength, or other logging parameters on a signed distance plot will show whether a fault is evidenced in that indicator dataset.

Signed distance plots also show if a fault is evidenced at any distance parallel to the surface being queried, and can be used to map, orient, and locate previously unmapped faults. Using a Python-based workflow, a plane can be quickly rotated through all possible orientations to locate and identify trends in a geotechnical 
dataset which may indicate faulting. The utility of these tools has been demonstrated in two open pit mines where faults important for slope stability assessments were identified and validated efficiently against large drilling datasets.

\section{References}

Bærentzen, JA \& Aanæs, H 2005, 'Signed distance computation using the angle weighted pseudonormal', IEEE Transactions on Visualization and Computer Graphics, vol. 11, no. 3, pp. 243-253, https://doi.org/10.1109/TVCG.2005.49

Barill, G, Dickson, NG, Schmidt, R, Levin, DIW \& Jacobson, A 2018, 'Fast winding numbers for soups and clouds', ACM Transactions on Graphics, vol. 37, no. 4, https://doi.org/10.1145/3197517.3201337

Choi, JH, Edwards, P, Ko, K \& Kim, YS 2016, 'Definition and classification of fault damage zones: a review and a new methodological approach', Earth-Science Reviews, vol. 152 (November 2017), pp. 70-87, https://doi.org/10.1016/j.earscirev.2015.11.006

CloudCompare 2019, version 2.10, computer software, http://www.cloudcompare.org

Danielson J, Stead D, Kinakin D \& Smith S 2017, 'The use of specific energy for fault mapping in an open pit mine', Proceedings of the 51st US Rock Mechanics/Geomechanics Symposium, American Rock Mechanics Association, Alexandria, pp. 815-821.

Danielson, J 2018, An Investigation into the Time Dependent Deformation Behaviour of Open Pit Slopes at Gibraltar Mine, BC, Canada, MSc thesis, Simon Fraser University, Burnaby.

Dawson-Haggerty, M 2019, Trimesh, Python library, https://github.com/mikedh/trimesh

Deere, DU \& Deere, DW 1988, 'The rock quality designation (RQD) index in practice', in L Kirkaldie (ed.), Rock Classification Systems for Engineering Purposes, STP 984, American Society for Testing Materials, Philadelphia, pp. 91-101.

Kington, J 2019, Mplstereonet, Python library, https://github.com/joferkington/mplstereonet

Paris, S \& Durand, F 2009, 'A fast approximation of the bilateral filter using a signal processing approach', International Journal of Computer Vision, vol. 81, pp. 24-52.

Pells, PJ, Bieniawski, ZT, Hencher, SR \& Pells, SE 2017, 'Rock quality designation (RQD): time to rest in peace', Canadian Geotechnical Journal, vol. 54, no. 6, pp. 825-834, https://dx.doi.org/10.1139/cgj-2016-0012

Richard, T, Dagrain, F, Poyol, E \& Detournay, E 2012, 'Rock strength determination from scratch tests', Engineering Geology, vol. 147-148, pp. 91-100, https://dx.doi.org/10.1016/j.enggeo.2012.07.011

Teale, R 1965, 'The concept of specific energy in rock drilling', International Journal of Rock Mechanics and Mining Sciences, vol. 2 , pp. 57-73. 
